\title{
USE OF DESIGN OF EXPERIMENTS TO OPTIMISE THE ENVIRONMENTAL PERFORMANCE OF CONSUMER PRODUCTS: A CASE STUDY OF SHOWERS AND TAPWARE IN NEW ZEALAND
}

\author{
M. Horrell ${ }^{\otimes}$, A. Shekar and S. McLaren \\ Massey University, New Zealand \\ $\triangle$ m.horrell@massey.ac.nz
}

\section{Abstract}

Eco-tools and techniques often lack guidance and a robust methodology for improving the environmental performance of products with an active use phase. A case study was undertaken to investigate the role of Design of Experiments (DoE) in providing insights to improve the environmental performance of two product categories with active use phases: showers and tapware. The results show how varying the components can reduce energy use and demonstrated how DoE can be used as an objective method for optimising a products environmental performance when user behaviour can influence the results.

Keywords: ecodesign, sustainable design, design process, active products, design of experiments

\section{Introduction}

Pressures from environmentally conscious consumers have led manufacturing companies to explore opportunities to develop products and systems with environmentally superior characteristics (Sousa and Wallace, 2006). This requires companies to identify relevant environmental criteria to guide the product development process (Sousa and Wallace, 2006), and use these criteria to assess alternative materials for components, modify the design so that it has an improved environmentally profile (Lenox, 2000), and/or create alternative products fulfilling the same function(s). To assist product developers in this process, a number of eco tools and methods are available including Design for the Environment (DfE) and Life Cycle Assessment (LCA), which can be used at various stages in New Product Development (NPD) (Fitzgerald et al., 2005). DfE tools, such as those based on checklists, are easy to implement but are very subjective and not particularly insightful in guiding improvements. Quantitative tools such as LCA are not only time consuming and costly, but tend to be unsuitable for early design processes due to the limited information available about the product at that stage in the design process (Devanathan et al., 2010). In particular, for companies developing products with an environmentally dominant use phase, the existing approaches are often inadequate. These types of products are described as "active products", and often the majority of environmental impacts are associated with energy consumption during the use stage of the product's life cycle (Jolliet et al., 2015). The task of addressing the future use phase in the design of a consumer product might be difficult for a company when there is more than one variable contributing to the environmental impacts associated with the use phase: these variables may not be obvious and need to be "discovered" by the designers. 
This discovery involves identifying the factors of the product's design (such as its shape, weight, interface) that affect the product's environmental impacts in its use phase. Furthermore, there may be a lack of empirical evidence that quantifies the environmental improvements when modifying a design to account for consumers' behaviour. As an example, a consumer survey study found a correlation between design factors of showers (i.e. spray type, colour, shape) and the user experience (Adeyeye et al., 2017). The study concluded that user experience and perceptions of the shower under different types of showerheads can significantly contribute to the use phase of the product - how enjoyable a product is for the user can determine how much it gets used. This suggests that companies need to develop an awareness of the factors influencing the environmental impacts associated with the use of their products in order to improve or innovate new products that do actually reduce overall environmental impacts (Herring and Roy, 2007).

Use of existing eco-tools has had limited relevance for active of products, as often these tools do not explicitly consider variability in how the user interacts (or potentially interacts) with the product or the influence of this behaviour on the magnitude of environmental impacts at the use stage (Wever et al., 2008). Generally existing environmental assessment approaches to support NPD (Renee et al., 2007, Boks and Stevels, 2003; Wever et al., 2008) either do not consider the environmental impacts related to human-product interactions or do so superficially, and there is a lack of guidance and robust methodologies for conducting this type of benchmarking (Lin et al., 2017). Studies which do address sustainable consumption tend to focus primarily on purchasing behaviours rather than the use of the product (Bhamra et al., 2011). This suggests that a new approach is needed to complement existing eco tools and provide companies with a practical method for addressing multiple variables (components, materials, user interfaces) that ultimately affect the environmental performance in the use phase of the product.

Design of Experiments (DoE) is one of the most popular benchmarking tools in product development since it commonly applied within Total Quality Management (TQM), an approach to support the improvement of manufacturing processes (Kutz, 2007). It has already been recognised as relevant in the context of environmental management as it can help minimise waste generation during the manufacturing phase of a product's life cycle (Kutz, 2007). A key component of TQM processes is some form of experimentation to test how the input variables from a device, component or manufacturing process affect the response variables that need to be optimised. DoE is considered to be the most effective experimentation tool when considering multiple input variables for a product system (Tanco et al., 2008; Roy, 2001). DoE avoids the misleading results that are often found in alternative experimentation methods such One Factor at a Time (OFAAT) and Randomised Control Trials (RCT), where the results only yield first order responses and ignore interactions between factors (Kutz, 2007). One study showed that most manufacturing companies applying DoE were those doing Research and Development projects (Tanco et al., 2008); however, the majority of manufacturing companies still apply inferior tools such as OFAAT (Granato and Ares, 2013). Kutz (2007) recommends that the DoE can also be used to optimise the environmental performance of a product, so the design is more useful and efficient in its use. This is supported by Gremyr et al. (2014) who emphasise that DoE used within the context of sustainable product development should aim to support the front end of product development by considering the full life cycle of the product, including the use phase. Furthermore, a case study has shown that DoE can be applied in a user-centred design to optimise a product's aesthetics, based on systematic statistical modelling of user-experience feedback from customers (Lin et al., 2017).

Inspired by Kutz (2007) and Gremyr et al. (2014), in this research a DoE industrial case study was undertaken to investigate improvement in the environmental performance of the use phase of an active consumer product, and provide insights into whether the DoE approach could be used more generally to support eco-design in New Product Development. A shower and tapware manufacturing company was selected for the case study. The company is based in Auckland, New Zealand; it has a considerable share of the market in New Zealand and Australia, and a growing presence in the United Kingdom and North America. The company sells a wide range of different shower and tapware products with varying spray technologies and user interfaces that are representative of variables that can be optimised to improve the environmental performance. Two individual DoE experiments were 
set up; (1) different shower products used in domestic household showers, and (2) various tap designs used in a corporate office bathroom. The process for designing and conducting the experiments followed four distinct stages: 'Generation of Ideas and Factors', "Development of case study", "Undertake case study" and "Interpretation of the results and analysis". The following sections follow this structure to describe the study and its results.

\section{Case study}

\subsection{Stage one: Generation of ideas}

An in-house seminar and practical workshop was attended by the engineers, designers and managers from the product development team. At the seminar, the researcher introduced the concept of Life Cycle Thinking and presented the results of an existing Life Cycle Assessment (LCA) study of a shower product (Institut Bauen und Umwelt, 2011) to show how the use phase dominates the environmental impacts for this product category. Teams brainstormed design factors that could influence the use phase of shower products, with a few of the generated design factors selected as appropriate to test in an experiment. They were chosen based on two aspects: ease of application and low risk in conducting the experiment. With respect to ease of application, the factors needed to be able to be relatively easily applied and tested in a household with minimal requirements for additional plumbing. With respect to risk, all equipment and components needed to be pressure rated to New Zealand plumbing standards and fitted professionally to the shower or bathroom to prevent permanent damage to the house from leaks or flooding. For the shower experiment the selected factors were: 'Spray Type' and 'Feedback to User'. For the tap experiment, the chosen factors were 'Cartridge Type' and 'Flow Rate'. In both experiments, the factors have levels representing the variations in the factor being tested (meaning all factors used are text-based rather than numeric). For the shower experiment, the factor 'Spray Type' had three levels: 'Luxury Spray A', 'Luxury Spray B' and 'Conventional'. The factor 'Feedback to User' had three levels: 'No Feedback', 'Usage Scale' and 'Monetary Cost'. For the tapware experiment, the factor 'Cartridge Type' had three levels: 'Progressive', '2-Step Eco' and 'Standard'. The factor 'Flow Rate' had two levels: '5 Litre' and '8 Litre'. These factors and levels are described in more detail in the following sections.

\subsection{Stage two: Development of case study}

The second stage of the process established a protocol for conducting the DoE experiments to identify and reduce nuisance variables. Two aspects were identified as significant to this experiment. A single shower unit can have multiple users where one user cannot clearly be distinguished from another one, so the response data may misrepresent the situation. Ideally it would be preferable to conduct the shower experiment using shower units that had only a single user. Similarly, the tapware experiment required that bathrooms with multiple basins would require all taps to follow the treatment order in parallel. The nuisance variables thought to be worthwhile including in the DoE and mitigating were ambient temperature and humidity in the shower room since this could impact the duration and temperature of the shower; however, due to excessive humidity saturation, the electronic sensor used to collect this data malfunctioned in some shower units and therefore these data had to be omitted from the final analysis. All shower units were pressure tested by the researcher prior to the experiment to ensure all had a high-pressure water supply (at least $200 \mathrm{kPa}$ ) to avoid misleading results from comparing low and high-pressure water systems. The purpose-built recording device (Figure 1) could be fitted between the shower hose and elbow of any rail system shower in a household. It recorded the primary response variables: Water Volume, Temperature, Duration and Energy on a flash memory card. Energy is calculated based on the thermal energy required to heat the volume of water per shower (based on the other response variables). These variables reflect what a shower and tap product consume when in use. For this calculation ambient temperature was assumed to be $15^{\circ} \mathrm{C}$. Water flow and temperature were recorded as analogue and digital signals. A real-time clock was included in the device to record duration of each shower event. This same device was adapted for the tapware experiment by adding an additional flowmeter, and temperature probe for the separate hot and cold 
inlets. Each unit was calibrated using a Center 370 RTD Temperature Probe and GPI Industrial Grade Electronic Flow Meter. As shown in Figure 1, the 'LCD Display' and 'Use Scale' were positioned in the shower to provide the user with information during their shower. The 'LCD display' can show numerical values while the 'Use Scale' has two sets of LED lights to indicate the low, medium and high use for energy (red) and water (blue).

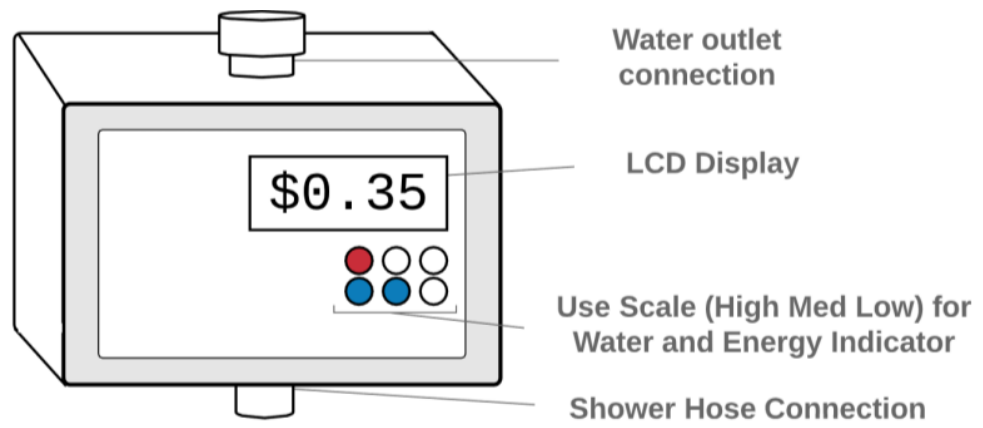

Figure 1. Purpose-built recording device

\subsection{Stage three: Undertake pilot studies}

\subsubsection{Experiment 1 - showers}

\section{Method}

The first task was to identify participants for the shower study. The use of social media and employee referrals from the case company generated a list of approximately 20 potential participants. However, finding willing participants who met the procedure requirement of a single user for the shower was difficult and somewhat unrealistic, and ultimately there were only two showers in the study that did not have multiple users. This resulted in monitoring and analysing five showers in different households that were used by a total of ten individuals. Participants were between the ages of 25 and 38 , and of low to medium household income. There were eight males and two females. Once the participants had been chosen, the researcher installed the datalogger into each household's shower. The general purpose of the experiment was explained to the participants; however, no further information was given to avoid influencing the results.

The multifactorial test design is shown in Table 1. Three types of existing spray types were used in the experiment. Luxury spray technology works by colliding water jets to form a larger coverage area with water droplets for a shower: Luxury Spray B provides greater coverage than that of traditional sprays, and Luxury Spray A does the same but uses a radically different method for creating the water droplets.

Table 1. Shower experiment factors and levels

\begin{tabular}{c|c|c} 
& Factor 1: Spray Type & Factor 2: Feedback to User \\
\hline Level 1 & Luxury Spray A & No Information \\
\hline Level 2 & Luxury Spray B & Energy and Water Usage (Low, Medium, High) \\
\hline Level 3 & Conventional & Cost Information
\end{tabular}

The 'Feedback to User' factor provided real-time information to the user about their current shower. Energy and water use were indicated using a low, medium and high LED light display referred to as the 'Use Scale'. Cost showed the user how much their shower cost in cents; additionally, when the shower was turned off a total cost for the month was shown. The cost calculation used a conservative estimation based on one of the lowest possible energy prices in New Zealand and assumed an 85\% energy efficiency of hot water heating and supply.

Every two weeks a new treatment was applied by returning to the household and changing the shower handset or adjusting the type of feedback that was presented to the user. This change was based on the 
randomised treatment order in Table 2. The duration between treatments allowed a sufficient number of showers to be recorded and averaged to compensate for the variability in the shower activities (e.g. washing hair) and multiple users who would likely have different showering habits.

Table 2. Treatment order for shower experiment

\begin{tabular}{|c|c|c|}
\hline StdOrder & Spray Type & Consumption \\
\hline 1 & Luxury Spray A & No Display \\
\hline 2 & Luxury Spray A & LED Display \\
\hline 3 & Luxury Spray B & LED Display \\
\hline 4 & Luxury Spray B & No Display \\
\hline 5 & Conventional & Cost Display \\
\hline 6 & Luxury Spray A & Cost Display \\
\hline 7 & Conventional & Cost Display \\
\hline 8 & Luxury Spray B & LED Display \\
\hline 9 & Conventional & \\
\hline
\end{tabular}

\section{Results}

The records of showers for all the households were averaged for each treatment. Figures 2 show the fitted means of all households for the response variable of interest. An Omnibus test was used to explain the variance to determine if a factor was statistically significant (where p-values $<0.05$ are considered significant). The baseline comparison is the first level of each factor. Figure 2 (A) shows the effect on Temperature for the factors 'Spray Type' and 'Display Type'. The 'Conventional Spray' had a lower Temperature than both the Luxury Sprays but none of the differences were found to be statistically significant. The 'Display Type' had a significant effect on Temperature with a p-value of 0.04. Compared to no feedback, the 'Cost' display was associated with a temperature increase of $0.14^{\circ} \mathrm{C}$ but this was not significant. The 'Use Scale' was associated with a temperature reduction of $0.48^{\circ} \mathrm{C}$ when compared to no display and this was significant (p-value 0.014). In summary, the UseScale influenced a reduction in temperature while the other factors and levels did not have a significant impact.

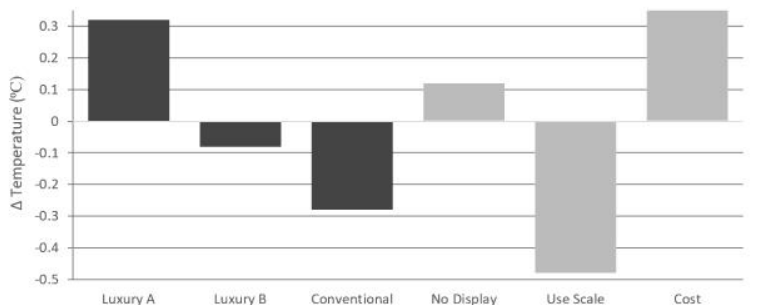

(A)

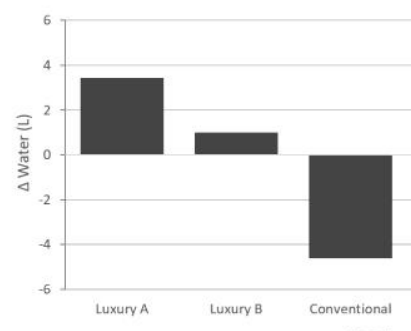

(C)

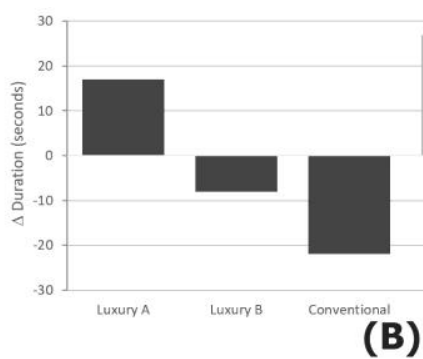

(B)

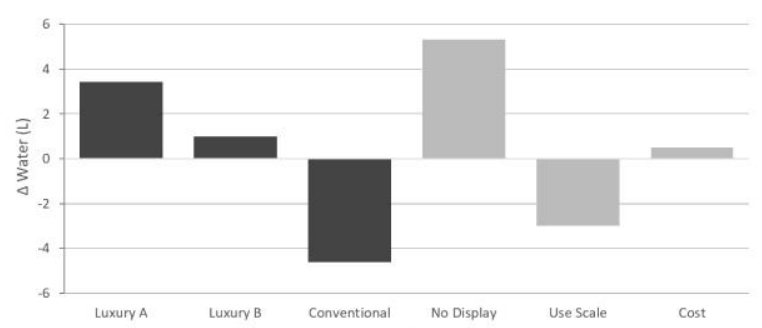

(D)

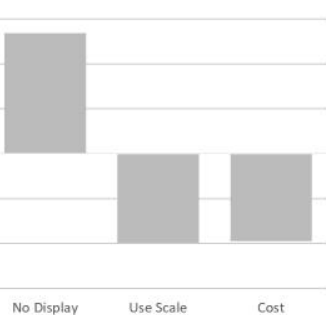

Figure 2. Showers - All factors response

Figure 2 (B) shows that, on average, the 'Conventional Spray' reduced the duration of the shower; however, this was not significant. An absence of any display was associated with an increased shower duration by 30.7 seconds and this was significant (p-value 0.036). Figure 2 (C) shows the response for 
Water. There was weak evidence (p-value 0.078) that spray type affected the total volume of water used per shower. The absence of a display indicated some increase in water usage compared to the Use-Scale and Cost factors however these were no statistically significant. Figure 2 (D) shows the response for Energy. 'Spray Type' was significant (0.049 p-value) while 'Display Type' was not. The Luxury-A showerhead was estimated to use $461 \mathrm{KJ}$ more than the mean of all showers ( $8 \%$ increase); however, this was not significant (0.061 p-value). There was a weak interaction between 'Luxury-A' and 'No-Display' with a p-value of 0.094 that estimated an increase of $580 \mathrm{KJ}$ of energy (10\% increase).

\subsubsection{Experiment 2 tapware}

\section{Method}

The tapware experiment was run in a corporate office in Auckland, New Zealand. A men's bathroom was selected that was used regularly by approximately 40 people. Recording devices were installed under two basins. Two taps were installed on to the basins; these taps were flexible in their design which enabled the researcher to change the internal and external components throughout the experiment.

The factors and levels chosen for this experiment are shown in Table 3.

Table 3. Tapware experiment factors and levels

\begin{tabular}{c|c|c} 
& Factor 1: Cartridge Type & Factor 2: Flow Restriction \\
\hline Level 1 & Progressive & $5 \mathrm{~L} / \mathrm{min}$ \\
\hline Level 2 & 2-Step Eco & $8 \mathrm{~L} / \mathrm{min}$ \\
\hline Level 3 & Standard
\end{tabular}

A cartridge is an internal component of a tap that allows for hot and cold water delivery to be varied depending upon the handle position. The standard cartridge has two degrees of freedom for delivery of water, which controls flow and temperature, while the progressive only has a single degree of freedom, which controls both flow and temperature. The design of the progressive means that hot water is only delivered when the handle is turned beyond $90^{\circ}$. The 2-Step Eco cartridge is a modified standard cartridge designed to reduce water use by having a distinct restriction of the movement of the handle when the flow has reached $50 \%$ of its maximum flow: users who require a higher flow must then apply a greater force to move the handle beyond this restriction.

Taps can be flow-restricted easily using a range of components from original equipment manufacturers (OEM). The flow restrictor can often impact the overall design of the tap as the body of the product may need to change to accommodate the size or shape of the component. In the tapware industry, it is generally assumed that lower flow restriction equates to reduced water consumption per use which is then reflected in most water saving and labelling schemes.

The multifactorial design and run order for the tapware experiment is shown in Table 4.

Table 4. Treatment order for tapware experiment

\begin{tabular}{c|c|c} 
StdOrder & Cartridge Type & Flow Restriction \\
\hline 1 & Progressive & $5 \mathrm{~L} / \mathrm{min}$ \\
\hline 2 & 2 -Step Eco & $5 \mathrm{~L} / \mathrm{min}$ \\
\hline 3 & Progressive & $8 \mathrm{~L} / \mathrm{min}$ \\
\hline 4 & Standard & $8 \mathrm{~L} / \mathrm{min}$ \\
\hline 5 & Standard & $5 \mathrm{~L} / \mathrm{min}$ \\
\hline 6 & 2-Step Eco & $8 \mathrm{~L} / \mathrm{min}$
\end{tabular}

The week before the experiment, all combination of tap modifications were introduced into the bathroom without recording. This was to avoid collecting data in the subsequent week where the users were experiencing the new design for the first time. For the experiment itself, a new combination was applied 
each day at approximately 12noon. Over six days 473 "washing hands" records were collected from all treatments. Since both hot and cold temperatures and flow were individually measured, it was not considered necessary to calculate energy use as it was assumed that the quantity of hot water used was representative of energy use. The response variables for the tap experiment were: Duration, Cold Water Use and Hot Water Use. An additional final response variable was calculated (PercentWaste) which represented any hot water that was drawn into the pipes but did not reach the tap and instead cooled to ambient temperature.

\section{Results}

The fitted means shown in the following graphs are the average of washing events for both taps for the response variable of interest. An Omnibus test was used to explain the variance to determine if a factor was statistically significant. Unless mentioned otherwise, no interactions between factors were found to be significant. Figure 3 (A) shows that the 'Cartridge Type' had no significant effect on the response Duration. However, there was weak evidence (p-value 0.181) that using a higher flow reduced Duration.

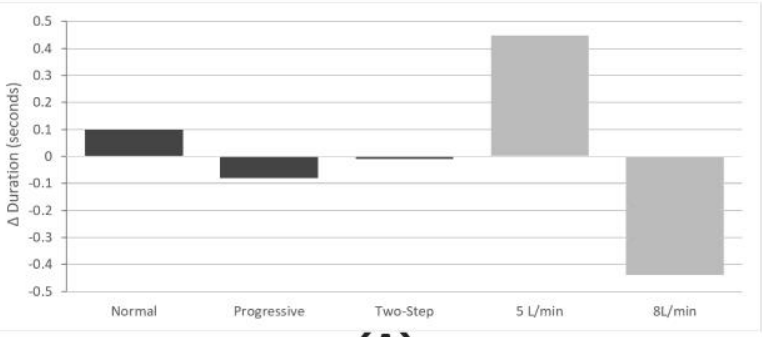

(A)

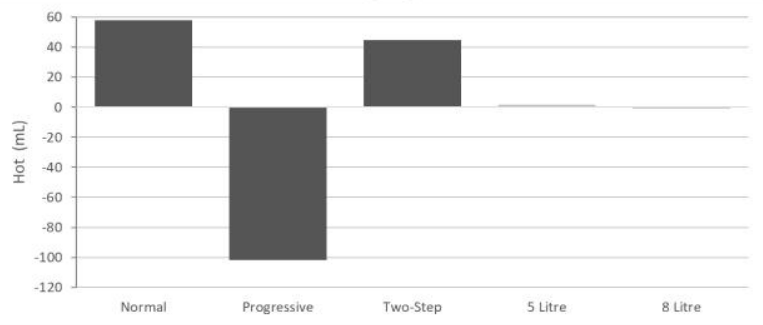

(C)

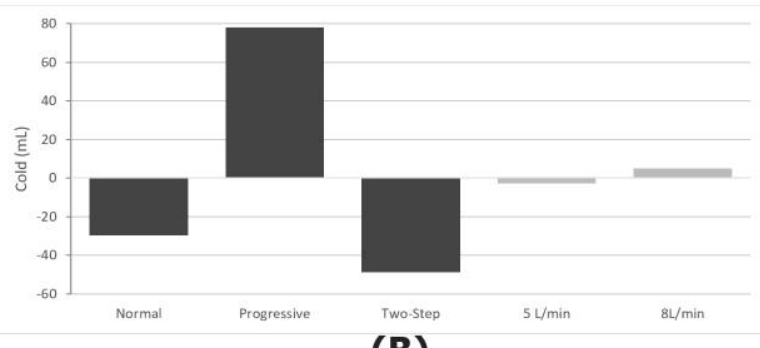

(B)
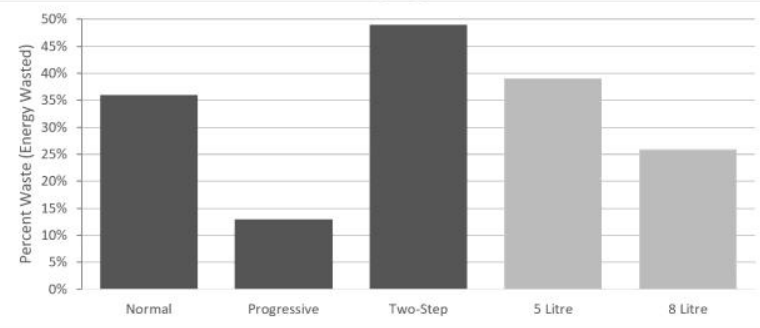

(D)

Figure 3. Tapware - All factors response

Flow had no statistically significant effect on Cold and Hot water use (Figure 3 C \& D). However, 'Cartridge Type' was statistically significant for both Cold and Hot water use. Using a Progressive cartridge was associated with an increase in cold water use by $78.1 \mathrm{~mL}$ (p-value 0.003 ) and decrease in hot water use by $102 \mathrm{~mL}$ (p-value < 0.001 ).

For PercentWaste (Figure 3 D), 'Cartridge Type' was statistically significant (p-value < 0.001). Flow was also found to be significant ( $\mathrm{p}$-value $<0.001$ ), but there was a significant interaction between Cartridge and Flow (p-value 0.040) which suggests the wasted energy (for heating water) increases when the cartridge distributes less hot water per washing hand event. This can be explained by the fact that a larger volume of hot water is more likely to travel through the pipes to the user than a smaller volume of water over the same time period.

\subsection{Stage four: Interpretation of results}

In the first experiment on showers, Spray type was shown to affect energy use of shower products while water volume, duration and temperature were not affected by Spray type. This finding poses a challenge to most water care rating schemes where it often recommends that a lower water star rating reduces hot water and associated energy costs. When displaying information to the user of a shower product, the type of display (or absence of a display) throughout the shower plays an important role. For this study, users responded positively by reducing their energy use when provided with information about whether they were a Low, Medium or High user (Use-scale display); in contrast, showing the monetary cost of the shower did not reduce the user's energy use. 
The second experiment presented some evidence that varying the flow restriction had no effect on the quantity of either hot or cold water used for washing hands. Instead, users washed for a longer or shorter time depending on the flow restrictor, effectively using the same amount of water per wash regardless of the flow restrictor. It was found that users will be less likely to wash with hot water with a Progressive Cartridge, and are more likely to waste hot water using the alternative Standard and 2-Step Cartridge since most users turn the handle to receive hot water, but do not wash long enough for the hot water to travel through the pipes and come out of the tap. This suggests that a Progressive Cartridge is environmentally superior as it wastes less energy based on the behaviours of users in this study.

\section{Discussion}

On the use of DoE for improving the environmental impacts of active products, firstly DoE provided the inherent ability to assess the behaviours of users and their related impact on the response variables. As an example, in the shower experiment there was evidence that information provided back to the user about their showering did influence their use of energy and, based on the type of feedback, it can produce a positive or negative response (displaying use information as a scale had a positive effect on energy use while showing the cost of the shower produced a negative effect on energy use). The tapware experiment demonstrated that varying components size, and position influenced the user's energy consumption. The DoE has therefore provided a method for understanding what changes can be made to the design of a new product when people and how they interact with the product is taken into consideration.

Secondly, DoE provides decision support when the subjective experiences and needs of different customers may impact the results and insights. Active products can be functional and experiential. As an example, the purpose of taking a shower can be classified as functional (i.e. getting clean) or relaxing (or both at the same time to varying degrees), and existing commercial products provide alternative spray types oriented towards one or other of these experiences. Simpler statistical tools often aggerate the all the results across participants which can lead to inaccurate results, however DoE addresses this by arranging experimental units into blocks (Blocking). Since different users or groups of users have different parameters for use, Blocking allows the practitioner to look at how those individual users respond to changing of the factors. To better inform designers in this area it would be advisable to include an additional response variable or a covariant that assesses the user's experience. For example, in the shower experiment a survey could have been used to capture this qualitative data. Thirdly, the practicality of conducting DoE in this way depends upon the product category. Producers need to be clear on what their objectives are for undertaking a DoE and the time required to do so. As an example, in the shower study it took a long time to collect the data as often only one data record could be recorded each day, while typically over 100 data records could be collected in the same time period for the tapware study. The implication is that some product categories are better suited for this type of experimentation than others i.e. those products that are used relatively frequently, and where user benefits are purely functional. Therefore, producers need to be aware of the practicality of varying DoE designs and match their expectations with realistic time horizons.

Validation of experiment results was difficult since most of the factors (Spray Type, User Feedback, Cartridge) analysed were non-numerical. At the end of a DoE experiment it is often necessary to translate the factorial design into the product development or manufacturing process to determine if the design is effective (Kutz, 2007). A DoE design that contains numeric factors allows the practitioner to develop a model for optimising the product design that reflects the ideal response based on varying all the factors and levels. This optimal product design could then be created and tested to validate the results of the DoE. Since this study applied non-numeric based factors mostly, it did not easily allow for this validation and this could diminish the usefulness of the results depending on how far along the product is within the NPD process. Furthermore, the decision support tools derived from this study are likely to be only relevant for a later stage of the development process, providing a method for benchmarking and varying the design of the product. To improve DoE for use in early design stages could be to further break down a part or technology to reflect its numerical components as additional factors. Using the shower study as an example, by replacing Spray Type with number of spray nozzles, size of the water jet and angle of the water collision it would allow the practitioner to 
identify a new spray type that has the least environmental impact. Its common practice for design engineers to create prototypes and trial them on customers to obtain formative feedback and adjust the design accordingly. Therefore, the DoE design could identify an optimum point where the experience is maximised, and the environmental impact is at its minimum.

\section{Conclusions}

Embedding environmental considerations into the product development process of active consumer products can be difficult for practitioners. This is particularly challenging when there are multiple factors to consider and where user behaviour may influence the environmental impacts associated with the product's life cycle. This study has demonstrated that DoE can be used as an objective method for obtaining new environmental insights in this area, and has led to recommendations for use of DoE in future experiments to support the NPD process. The factors and levels in a DoE experiment need to be carefully selected to reflect the stage of the product development process or type of NPD project. NPD projects can typically be categorised as radical or incremental (Slater et al., 2014). In the context of a shower product, a radical project could be the development of a new spray technology where the product developer can use modelling and prototyping techniques in the early stages of development to modify the waterjet size, amount and speed. Since these are variables that could ultimately impact the use phase of the product, there is an opportunity to implement DoE using numerical factors to optimise the design for active use. However, when implementing DoE in an incremental project it may be more appropriate to use text-based factors and levels. In this type of project, the objective is to provide an environmental point of reference and comparison of products, components or technology; this comparison may be used to support material and part selection during the later stage of the NPD process when the design specifications are being formalised. Common decision support tool for material and part selection implemented during the NPD process are Pugh Charts and Weight Factor Matrix (Otto and Wood, 2001, Ulrich and Eppinger, 2012).

In the shower study, the attitudes or feelings of the participants were not recorded as each combination of factors and levels were tested. This led to a realisation that it is important to include qualitative data in the DoE design when the user benefits of the product are not purely functional. This could involve recording response variables that are both quantitative (energy use of the prototype) and qualitative (experience of the customer) so that the final analysis can include consideration the correlation or trade-offs between them. One of the obvious limitations of the study is the small sample of active product categories. Therefore, the implications for the use of DoE to inform the design process may not be representative of all active products.

\section{Acknowledgements}

This research is funded and supported by Callaghan Innovation [grant number MTVN1503]

\section{References}

Adeyeye, K., She, K. and Baïri, A. (2017), "Design factors and functionality matching in sustainability products: A study of eco-showerheads", Journal of Cleaner Production, Vol. 142 No. Part 4, pp. 4214-4229. https://doi.org/10.1016/j.jclepro.2016.11.143

Bhamra, T., Lilley, D. and Tang, T. (2011), "Design for Sustainable Behaviour: Using Products to Change Consumer Behaviour", Design Journal, Vol. 14 No. 4, pp. 427-445. https://doi.org/10.2752/17563061 $1 \mathrm{X} 13091688930453$

Boks, C. and Stevels, A. (2003), "Theory and practice of environmental benchmarking in a major consumer electronics company”, Benchmarking: An International Journal, Vol. 10 No. 2, pp. 120-135. https://doi.org/ $10.1108 / 14635770310469653$

Devanathan, S. et al. (2010), "Integration of sustainability into early design through the function impact matrix", Journal of Mechanical Design, Transactions of the ASME, Vol. 132 No. 8, pp. 0810041-0810048. https://doi.org/10.1115/1.4001890

Fitzgerald, D.P. et al. (2005), University of Maryland, College Park, MD 20742, USA.

Granato, D. and Ares G. (2013), Mathematical and statistical methods in food science and technology: Chichester, West Sussex, UK : John Wiley \& Sons Inc. 
Gremyr, I. et al. (2014), "Adapting the Robust Design Methodology to support sustainable product development", Journal of Cleaner Production, Vol. 79, pp. 231-238. http://dx.doi.org/10.1016/j.jclepro. 2014.05.018

Herring, H. and Roy, R. (2007), "Technological innovation, energy efficient design and the rebound effect", Technovation, Vol. 27, pp. 194-203. https://doi.org/10.1016/j.technovation.2006.11.004

Institut Bauen und Umwelt e.V. (2011), Environmental Product Declaration as per ISO 14025 for hand showers Croma 100 and Crometta 85. Panoramastraße, Berlin.

Jolliet, O. et al. (2015), "Inventory Analysis of Emissions and Extractions", In Environmental Life Cycle Assessment, Vol. 57. CRC Press.

Kutz, M. (2007), Environmentally conscious mechanical design, Wiley series in environmentally conscious engineering: Hoboken, NJ: John Wiley.

Lenox, M. (2000), "An Assessment of Design-for-Environment Practices in Leading US Electronics Firms", Interfaces, Vol. 30 No. 3, pp. 83-94.

Lin, K.Y. et al. (2017), "User-experience-based design of experiments for new product development of consumer electronics and an empirical study", Journal of Industrial and Production Engineering, Vol. 34 No. 7, pp. 504-519. https://doi.org/10.1080/21681015.2017.1363089

Otto, K.N. and Wood, K.L. (2001), Product design : techniques in reverse engineering and new product development: Upper Saddle River, NJ : Prentice Hall.

Renee, W. et al. (2007), "Increasing the benefits of product-level benchmarking for strategic eco-efficient decision making," Benchmarking: An International Journal Vol. 6, p. 711. https://doi.org/10.1108/ 14635770710834509.

Roy, R.K. (2001), Design of experiments using the Taguchi approach : 16 steps to product and process improvement, John Wiley \& Sons, New York ; Chichester.

Slater, S.F., Mohr, J.J. and Sengupta, S. (2014), "Radical product innovation capability: Literature review, synthesis, and illustrative research propositions", Journal of Product Innovation Management, Vol. 31 No. 3, pp. 552-566. https://doi.org/10.1111/jpim.12113

Sousa, I. and Wallace, D. (2006). "Product classification to support approximate life-cycle assessment of design concepts," Technological Forecasting and Social Change, Vol. 73 No. 3, pp. 228-249. http://dx.doi.org/10. 1016/j.techfore.2004.03.007.

Tanco, M. et al. (2008), "Is design of experiments really used? A survey of Basque industries", Journal of Engineering Design, Vol. 19 No. 5, pp. 447-460. https://doi.org/10.1080/09544820701749124

Ulrich, K.T. and Eppinger, S.D. (2012), Product design and development: New York: McGraw-Hill/Irwin.

Wever, R., van Kuijk, J. and Boks, C. (2008), "User-centred design for sustainable behaviour", International Journal of Sustainable Engineering, Vol. 1 No. 1, pp. 9-20. https://doi.org/10.1080/19397030802166205 